\title{
BANACH SPACES WITH AN UNCONDITIONAL BASIS THAT ARE ISOMORPHIC TO A NONATOMIC BANACH LATTICE
}

\author{
LEA MCCLARAN
}

(Communicated by Dale Alspach)

\begin{abstract}
It is proved that if $X$ is a Banach space with an unconditional basis, then $X$ is isomorphic to a nonatomic Banach lattice which is not order continuous if and only if $X$ contains a subspace isomorphic to $c_{0}$.
\end{abstract}

Finite Borel measures on separable, complete metric spaces come in three types: Legesgue measure on $[0,1]$ (nonatomic measures), counting measure on the integers (atomic measures), and a combination of the first two [R]. It is the same with Banach lattices. An atom in a Banach lattice is an element $a>0$ such that for all $b>0$ with $a>b, b$ is a scalar multiple of $a$. Purely atomic (discrete) Banach lattices are those for which every positive element dominates some atom. Purely nonatomic (continuous) Banach lattices are those that have no atoms. So, Banach lattices, like nice measures, come in three types: the purely atomic ones, the purely nonatomic ones, and those that are a sum of the two. The question considered in this paper is: when are purely atomic Banach lattices isomorphic to purely nonatomic ones? The scope of this paper is to consider only purely atomic lattices with an unconditional basis giving the lattice structure. That is, the atoms are the basis elements and positive multiples thereof.

Recently, Kalton and Wojtaszczyk [KW] characterized those Banach spaces with an unconditional basis which are isomorphic to order-continuous, (purely) nonatomic Banach lattices. An order-continuous Banach lattice is one where every downwardly directed net which converges in order to 0 also converges in norm to 0. Additionally, it is known that every lattice structure on a Banach lattice not containing $c_{0}$ is order-continuous [LT2, pp. 6-8]. [KW] showed that a Banach lattice $X$ with unconditional basis is isomorphic to a nonatomic ordercontinuous Banach lattice if and only if $X \approx X\left(l_{2}\right)$. However, their paper does not address the question of when a space is isomorphic to a nonatomic Banach lattice which is not order-continuous. Since E. Lacey and P. Wojtaszczyk [LW]

Received by the editors May 17, 1993.

1991 Mathematics Subject Classification. Primary 46B42.

Supported in part by NSF \#DMS89-21369.

The results of this paper are contained in the author's doctoral dissertation being prepared at Texas A\&M University under the supervision of W. B. Johnson. 
showed that $c_{0}$ is isomorphic to a nonatomic, not order-continuous Banach lattice; one might guess that $X$ has the same property if and only if $X \approx$ $X\left(c_{0}\right)$. While it is certainly true that $X\left(c_{0}\right)$ is isomorphic to a nonatomic, not order-continuous Banach lattice, the necessity is false. As an example, consider $X=l_{2} \oplus c_{0}$; this $X$ is isomorphic to a nonatomic, not order-continuous, Banach lattice, since both $\iota_{2}$ and $c_{0}$ are, but $X \not \approx X\left(c_{0}\right)$.

Before the paper [KW], it was known [AW] that $l_{1}$ is not isomorphic to a nonatomic Banach lattice while $l_{2}$ and (by [LW]) $c_{0}$ are. So it is interesting to consider the cases of

$$
l_{1} \oplus l_{2} \quad \text { and } \quad l_{1} \oplus c_{0}
$$

The results of [KW] imply that $l_{1} \oplus l_{2}$ is not isomorphic to a nonatomic Banach lattice, since it does not contain $c_{0}$ and hence every lattice structure on it is order-continuous. A surprising consequence of Theorem 1 (below) is that $l_{1} \oplus c_{0}$ is isomorphic to a nonatomic, not order-continuous Banach lattice. So there is something special in the structure of $c_{0}$ that $l_{2}$ does not have. This paper characterizes those Banach spaces with an unconditional basis which are isomorphic to nonatomic Banach lattices which are not order-continuous.

Theorem 1. Let $X$ be a Banach space with an unconditional basis. Then $X$ is isomorphic to a nonatomic Banach lattice which is not order-continuous if and only if $X$ contains a subspace isomorphic to $c_{0}$.

The necessity is clear from the fact that Banach lattices not containing $c_{0}$ are order-continuous. The proof of sufficiency consist of the construction of a nonatomic, not order-continuous Banach lattice $Y$ and then several lemmas that show that $X \approx Y$.

\section{CONSTRUCTION}

The space $Y$ is based on the construction by E. Lacey and P. Wojtaszczyk [LW] of a nonatomic Banach lattice isomorphic to $c_{0}$. In [LW], the desired space is a certain collection of continuous functions on a certain compact space $T$, under the sup norm. Our construction will use the same space $T$ but modify the collection of continuous functions; our norm depends on $X$ and is the sup norm when $X=c_{0}$.

$T$ is a space of closed intervals in the first quadrant of the plane. These intervals are built in levels, and each of the intervals originate at the point $(0,0)$. To construct the intervals, first pick a number $0<r<1$. Let $I_{0}$ be the unit interval along the $y$-axis. This is the 0th level. For the first level, pick a sequence of intervals (originating at $(0,0)$ ) of length $r$ whose slopes increase to $+\infty$. Call these intervals $I_{0, n}$, where $n$ increases with the slopes of the intervals. On the second level, for each $n$, pick a sequence of intervals of length $r^{2}$ whose slopes converge to the slope of $I_{0, n}$, and which lie between $I_{0, n}$ and $I_{0, n-1}$ whenever $n>1$. Continue in this manner, so that at the $n$th level we have sequences of intervals of length $r^{n}$. The compact set $T$ is the union of all of these intervals. (See Figure 1.)

Now let $S_{n}$ be the part of the circle $x^{2}+y^{2}=\left(r^{n}\right)^{2}$ that lies in the first quadrant. Let $\left\{w_{i}\right\}_{i=1}^{\infty}=\left(S_{1} \cap T\right) \backslash\{(0, r)\}$. Let $\left\{e_{i}\right\}_{i=1}^{\infty}$ be the unconditional 


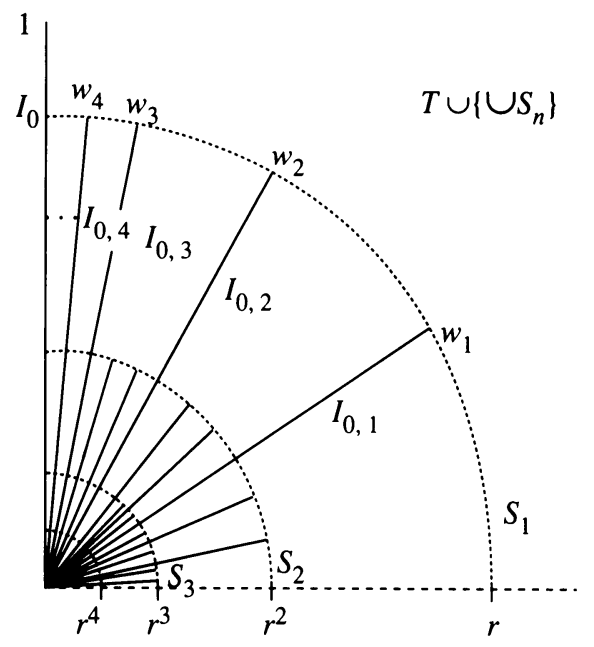

FIGURE 1

basis for $X$. We define the space $Y$ by

$$
\begin{aligned}
Y=\{f \in C(T) & : f(0, \alpha)=0 \text { for } 0 \leq \alpha \leq 1, \\
& \left.f \text { is affine on intervals, } \sum_{i} f\left(w_{i}\right) e_{i} \in X\right\} .
\end{aligned}
$$

Let $\|\cdot\|_{Y}$ be defined for $f \in Y$ by

$$
\|f\|_{Y}=\max \left\{\left\|\sum_{i} f\left(w_{i}\right) e_{i}\right\|_{X}, \sup _{t \in T}|f(t)|\right\},
$$

and let the order on $Y$ be the natural order; that is, $f \leq g$ if and only if $f(t) \leq$ $g(t)$ for all $t \in T$. The following is a fact about spaces with unconditional bases that we will use often.

Lemma 2. For each $w_{k} \in S_{1} \cap T$ and for each $f \in Y,\left|f\left(w_{k}\right)\right| \leq\left\|\sum_{i} f\left(w_{i}\right) e_{i}\right\|_{X}$. Proof. See [LT1, Proposition 1.c.7].

It is easy to see that $Y$ is a Banach lattice when endowed with the norm and order given above and when you consider that $X$ is a Banach lattice with the order given by its unconditional basis $\left\{e_{i}\right\}_{i=1}^{\infty}$. To see that $Y$ is nonatomic, consider a function $0<f \in Y$. Recalling that $f$ is continuous on $T, f$ is affine on intervals, and every interval in $T$ has an initial segment that is the limit of other, shorter intervals, we see that $f$ cannot be supported entirely on only one interval. Take intervals $I_{1}$ and $I_{2}$ in the support of $f$. Clearly, it is possible to choose $0<g \in Y$ so that $g<f, g=1 / 2 f$ on $I_{1}$, and $g=1 / 3 f$ on $I_{2}$. This function $g$ is less than $f$ but not a multiple of $f$, hence $f$ is not an atom.

Now for each $s \in S_{n}$, either $s \in T \cap S_{n}$, or there are closest $s^{\prime}$ and $s^{\prime \prime}$ in $T \cap S_{n}$ such that $\arg s^{\prime}<\arg s<\arg s^{\prime \prime}$. Let $J_{s}$ be the interval connecting 


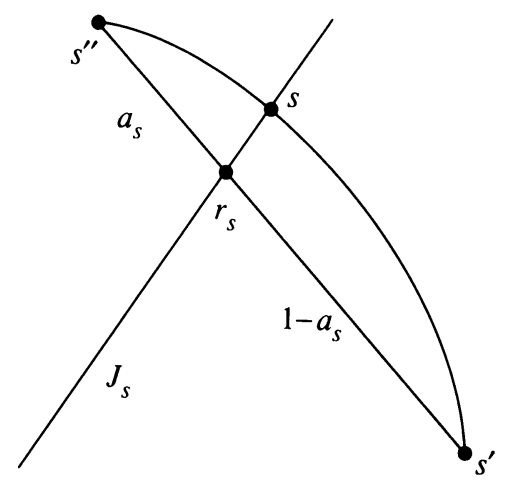

FIGURE 2

$s$ to the point $(0,0)$, and let $r_{s}$ be the intersection of $J_{s}$ with the chord connecting $s^{\prime}$ and $s^{\prime \prime}$. Then there is a number $a_{s}$ with $0<a_{s}<1$ such that $r_{s}=a_{s} s^{\prime}+\left(1-a_{s}\right) s^{\prime \prime}$. (See Figure 2.)

For $f \in Y$ and $s \in S_{n}$, define

$$
\Phi_{n}(f)(s)=a_{s} f\left(s^{\prime}\right)+\left(1-a_{s}\right) f\left(s^{\prime \prime}\right)
$$

with $a_{s}, s^{\prime}, s^{\prime \prime}$ defined as above. Then $\Phi_{n}: Y \rightarrow C\left(S_{n}\right)$ is an extension operator; that is, $\Phi_{n}$ takes $\left.f\right|_{S_{n} \cap T}$ and extends it to a continuous function on $S_{n}$ without increasing $\sup |f|$. Define $Q_{n}: Y \rightarrow Y$ by

$$
Q_{n}(f)(t)=\left(|t| / r^{n}\right) \Phi_{n}(f)\left(r^{n} t /|t|\right) .
$$

$Q_{n}$ is a projection on $Y . Q_{n}(Y)$ is the collection of functions on $Y$ which are completely determined by their values on $T \cap S_{n}$.

Lemma 3. $\left\|Q_{n}\right\|=1$.

Proof. Let $f \in Y$ with $\|f\|=1$. Then $\left\|Q_{n} f\right\|_{Y}=\max \left\{\left\|\sum_{i} Q_{n} f\left(w_{i}\right) e_{i}\right\|_{X}\right.$, $\left.\max _{t}\left|Q_{n} f(t)\right|\right\}$. If $t \in T$ with $|t|<r^{n}$, then

$$
\left|Q_{n} f(t)\right|=\frac{|t|}{r^{n}}\left|\Phi_{n}(f)\left(\frac{r^{n} t}{|t|}\right)\right| \leq 1
$$

because $|t| / r^{n}<1$ and $|f(t)| \leq 1$ for all $t$. If $1 \geq|t| \geq r^{n}$, then $r^{n} t /|t| \in S_{n} \cap T$ so that $\Phi_{n}(f)(t)=f(t)$. Hence,

$$
\left|Q_{n} f(t)\right|=\frac{|t|}{r^{n}}\left|f\left(\frac{r^{n} t}{|t|}\right)\right|=|f(t)| \leq 1 .
$$

Finally,

$$
\left\|\sum_{i} \frac{r}{r^{n}} \Phi_{n} f\left(\frac{r^{n} w_{i}}{r}\right) e_{i}\right\|=\left\|\sum_{i} \frac{r}{r^{n}} f\left(\frac{r^{n} w_{i}}{r}\right) e_{i}\right\|=\left\|\sum_{i} f\left(w_{i}\right) e_{i}\right\| \leq 1 .
$$

Lemma 4. $\lim _{n \rightarrow \infty}\left\|Q_{n} f-f\right\|=0$ for any $f \in Y$.

Proof. The previous two lemmas and the definition of $Q_{n}$ show that if $t \in T$ and $|t| \geq r^{n}$, then $Q_{n} f(t)=f(t)$ and if $|t|<r^{n}$, then $\left|Q_{n} f(t)\right| \leq$ $\max _{s \in S_{n} \cap T}|f(s)|$. Let $f \in Y$ and let $\varepsilon>0$. Since $f$ is continuous at $(0,0)$, 
we can choose $N$ so large that if $|t| \leq r^{N}$, then $|f(t)|<\varepsilon$. Now $Q_{n} f(t)=f(t)$ whenever $|t| \geq r^{n}$. So if $n>N$, then

$$
\left\|\sum_{i}\left(Q_{n} f\left(w_{i}\right)-f\left(w_{i}\right)\right) e_{i}\right\|=0
$$

and

$$
\max _{t}\left|Q_{n} f(t)-f(t)\right| \leq \max _{t \in S_{n} \cap T}|f(t)|+\sup _{|t|<r^{N}}|f(t)| \leq 2 \varepsilon,
$$

so that $\left\|Q_{n} f-f\right\|<2 \varepsilon$.

It is easy to see that $Q_{n} Q_{m}=Q_{\min (n, m)}$. If we let $Q_{0}=0$, then the above facts show that $Y=\sum_{n}\left(Q_{n}-Q_{n-1}\right)(Y)$. If we let $Y_{n}=\left(Q_{n}-Q_{n-1}\right)(Y)$, then it is easy to see that $Y_{n}$ is the set of all $f \in Y$ such that $f$ is completely determined by its values on $S_{n}$ and $f=0$ on intervals of length greater than $r^{n}$. Hence, if we give $Y_{1}$ the natural norm inherited from $X$ and give $Y_{n}$ the sup norm for each $n$, then $Y_{1}=Q_{1}(Y) \approx X, Y_{2}=\left(\sum \oplus c_{0}\right)_{c_{0}}=c_{0}$, and $Y_{n}=\left(\sum \bigoplus Y_{n-1}\right)_{c_{0}}=\left(\sum \bigoplus c_{0}\right)_{c_{0}}$ for $n>2$.

Next, define a mapping $T$ taking $Y$ to $\left(\sum_{n} \oplus Y_{n}\right)_{c_{0}}$ by $T(f)=\left(Q_{1}(f)\right.$, $\left.\left(Q_{2}-Q_{1}\right) f,\left(Q_{3}-Q_{2}\right) f, \ldots\right) . T$ is a bounded linear operator since

$$
\begin{aligned}
\|T f\| & =\max \left\{\left\|Q_{1} f\right\|,\left\|\left(Q_{2}-Q_{1}\right) f\right\|,\left\|\left(Q_{3}-Q_{2}\right) f\right\|, \ldots\right\} \\
& \leq \max \{\|f\|, 2\|f\|, 2\|f\|, \ldots\} \leq 2\|f\| .
\end{aligned}
$$

Lemma 5. $T^{-1}$ is bounded.

Proof. $T^{-1}\left(f_{1}, f_{2}, f_{3}, \ldots\right)=\sum_{n} f_{n}$. The proof that $\sum_{n} f_{n}$ is defined is contained in the proof that $T^{-1}$ is bounded. Now

$$
\left\|T^{-1}\left(f_{1}, f_{2}, f_{3}, \ldots\right)\right\|=\left\|\sum_{n} f_{n}\right\|=\max \left\{\left\|\sum_{i} f_{1}\left(w_{i}\right) e_{i}\right\|_{X}, \max _{t}\left|\sum_{i} f_{i}(t)\right|\right\} .
$$

Clearly, $\left\|\sum_{i} f_{1}\left(w_{i}\right) e_{i}\right\|_{X} \leq\left\|\left(f_{1}, f_{2}, f_{3}, \ldots\right)\right\|$. For the rest, let $t \in T$ be fixed. Then there is a largest $k$ such that $t$ belongs to an interval of length $r^{k}$. So, for this $t$

$$
\left|\sum_{i} f_{i}(t)\right| \leq \sum_{i}\left|f_{i}(t)\right| \leq \sum_{i=1}^{k}\left|f_{i}(t)\right|
$$

since $f_{i} \equiv 0$ on any interval of length greater than $r^{i}$. Now for each $i$, since $|t| \leq r^{k} \leq r^{i}$ and $f$ is affine on intervals,

$$
\left|f_{i}(t)\right|=\frac{|t|}{r^{i}}\left|f_{i}\left(\frac{t}{|t|} r^{i}\right)\right| \leq r^{k-i} \sup _{s \in S_{i} \cap T}\left|f_{i}(s)\right| .
$$

Thus, if $i>1,\left|f_{i}(t)\right| \leq r^{k-i}\left\|f_{i}\right\|$ and if $i=1$, we may apply Lemma 2 to get $\left|f_{i}(t)\right| \leq r^{k-i}\left\|f_{i}\right\|$. Hence,

$$
\begin{aligned}
\sum_{i=1}^{k}\left|f_{i}(t)\right| & \leq \sum_{i=1}^{k}\left\|f_{i}\right\| r^{k-i} \leq\left\|\left(f_{1}, f_{2}, f_{3}, \ldots\right)\right\| \sum_{j=0}^{\infty} r^{j} \\
& \leq\left\|\left(f_{1}, f_{2}, f_{3}, \ldots\right)\right\| \frac{1}{1-r},
\end{aligned}
$$

so that $T^{-1}$ is bounded. 
This shows that $Y \approx\left(\sum_{n} \oplus Y_{n}\right)_{c_{0}} \approx X \oplus \sum_{n} c_{0} \approx X \oplus c_{0}$. The fact that $Y$ is not order-continuous is easy to see from its construction ( $Y$ contains lattice copies of $c$ ) or from the fact that the only order-continuous order on $c_{0}$ is the canonical one [AW], and $c_{0}=\left(\sum_{n=2}^{\infty} \oplus Y_{n}\right)_{c_{0}}$ does not have the canonical order. The following well-known lemma will finish the proof of the theorem.

Lemma 6. $X \approx X \oplus c_{0}$.

Proof. Since $X$ contains $c_{0}$, then $X$ contains $c_{0}$ complementably (see [LT1, 2.f.5]). Thus, $X \approx Y \oplus c_{0}$ for some $Y \subset X$. However, $c_{0} \approx c_{0} \oplus c_{0}$ so that $X \approx Y \oplus\left(c_{0} \oplus c_{0}\right) \approx\left(Y \oplus c_{0}\right) \oplus c_{0} \approx X \oplus c_{0}$.

\section{REFERENCES}

[AB] C. D. Aliprantis and O. Burkinshaw, Positive operators, Academic Press, New York, 1984.

[AW] Ju. A. Abramovič and P. Wojtasczcyk, On the uniqueness of order in the spaces $l_{p}$ and $L_{p}[0,1]$, Mat. Z. 18 (1975), 313-325.

[KW] N. J. Kalton and P. Wojtasczcyk, On nonatomic Banach lattices and Hardy spaces, Proc. Amer. Math. Soc. 120 (1994), 731-741.

[LW] H. E. Lacey and P. Wojtaszczyk, Nonatomic Banach lattices can have $l_{1}$ as a dual space, Proc. Amer. Math. Soc. 57 (1976), 79-84.

[LT1] J. Lindenstrauss and L. Tzafriri, Classical Banach spaces I, Sequence spaces, Springer-Verlag, Berlin, 1977.

[LT2] __ Classical Banach spaces II, Function spaces, Springer-Verlag, Berlin, 1979.

[R] H. Royden, Real analysis, Macmillan, New York, 1988.

Department of Mathematics, TeXas A\&M University, College Station, TeXas 778433368

E-mail address: Lea.McClaran@tamu.edu 